\title{
Modeling and Simulation of Embryonic Hardware Structures Designed on FPGA-based Artificial Cell Network Topologies
}

\author{
Cs. Szász, V. Chindriş, L. Szabó \\ Department of Electrical Engineering \\ Technical University of Cluj, 400020, Romania \\ e-mail: Csaba.Szasz@edr.utcluj.ro
}

\begin{abstract}
The cell-based structure, which makes up the majority of biological organisms, involve nearly perfect selforganization, self-reproduction (multiplying), and faulttolerance properties in a well organized hierarchical mechanism. By adapting these mechanisms and capabilities from nature, scientific approaches have helped researches understand related phenomena and associated with principles to engine complex novel digital systems and improve their capability. Founded by these observations, the paper is focused on computer-aided modeling and simulation of embryonic hardware configurations designed on FPGA-based artificial cell network topologies. Own developed artificial cell model and artificial organism models are proposed, as basic components of a four level embryonic hardware structures. These models are careful tested through computer-aided investigations, using a specially developed software toolkit designed for VLSI systems real-time simulation operations.
\end{abstract}

\section{INTRODUCTION}

As well-known, the latest generations of VLSI (Very Large Scale Integrated) circuit's-based digital systems are characterized by highly computational and processing power, implemented on complex hardware architectures. Beside of its huge benefits in the control solutions implementation, unfortunately they introduce for designer engineers also a lot of difficulties booth in the fault detection and fault elimination operations [1], [2].

The solution from all these inconveniences seems to be offered by researchers from microelectronic sciences which have early discovered, that, by adopting selfhealing and surviving mechanisms of biological organisms from nature, becomes possible to design complex novel digital systems provided with highly faulttolerance and robustness properties. The basic idea is to imitate the biological organism's cell-based structure which involve nearly perfect self-organization, selfreproduction (multiplying), and fault-tolerance properties in a well organized hierarchical mechanism. Focusing on this strategy, it is expected to implement VLSI hardware structures able to simulate biological cells or artificial organism basic functions in similar mode with their equivalents from the living world [3], [4].

All these research efforts are based on a new concept born on digital systems design: the embryonic systems.
For this reason, basic concepts from cellular embryonic theory can be used for VLSI digital systems design. Therefore terms like artificial molecule, artificial cell and artificial organism are key concepts in developing and implementing embryonic hardware systems [5], [6].

\section{EMBRYONIC SYSTEMS DEVELOPMENT STRATEGIES}

The international research efforts in bio-inspired hardware systems (embryonic science) are very intensive and dynamic. This large interest is caused by the big problems in the more complex VLSI circuits manufacturing processes, which involve difficulties for designer engineers (manufacturing errors, circuits size, fault tolerance, etc.), and through the large impact in informatics an microelectronics technologies development (VLSI circuits minimization, nanotechnology scale circuits design and manufacturing).

At the international level, two basic models are developed for the bio-inspired digital embryonic systems: architecture with two-level embryonic structures, developed at University of York, England [1], [2], and architecture with four-level embryonic structures developed at the Swiss Federal Institute of Technology [3], [4].

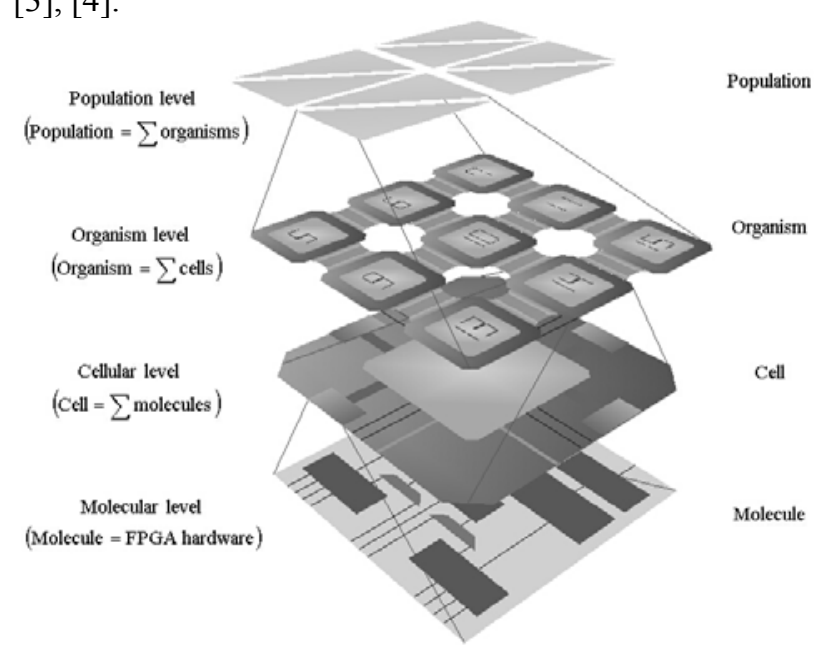

Fig. 1. The four level of an embryonic system

Taking into account the differences between real world, biological and digital systems, the Logic Systems Laboratory from Swiss Federal Institute of Technology, 
EPFL Lausanne, developed a bio-inspired system architecture, founded on four level of organizing, similar to figure 1 [4], [5]. This is a POE-type (Phylogeny, Ontogeny and Epigenesis) embryonic system developed in analogy with the evolutionary processes of biological systems. In accordance with this model, these embryonic systems derive from the multi-cellular structure of complex living organisms with strong hierarchical organization from molecular to population levels. The POE classification was introduced at first time by the researcher E. Sanchez for the digital electronic systems classification [4]. It is important to note, that the only hardware model of the above mentioned POE-type systems is the basic located molecular level [4], [6].

A main conceptual difference regarding the classical POE-type embryonic structures implementation is expressed in figure 1, where is proposed a solution in which all 4 embryonic levels can be implemented as a novel digital FPGA (Field Programmable Gate Array).

\section{FPGA-BASED ARTIFICIAL CELL MODEL FOR EMBRYONIC SYSTEMS DEVELOPMENT}

Considering the observations made in the previous chapter, the paper presents some research efforts regarding the development of an FPGA-based artificial cell model with a generalized character, in order to reproduce with high fidelity the complex phenomena and interaction rules inside embryonic hardware systems. For this reason, an original structure for the artificial cell is proposed, as shown in figure 2. According to this model, each FPGA circuit is considered an artificial cell with 4 lattices capable of full operation in a network structure through specially defined data and control buses [7].

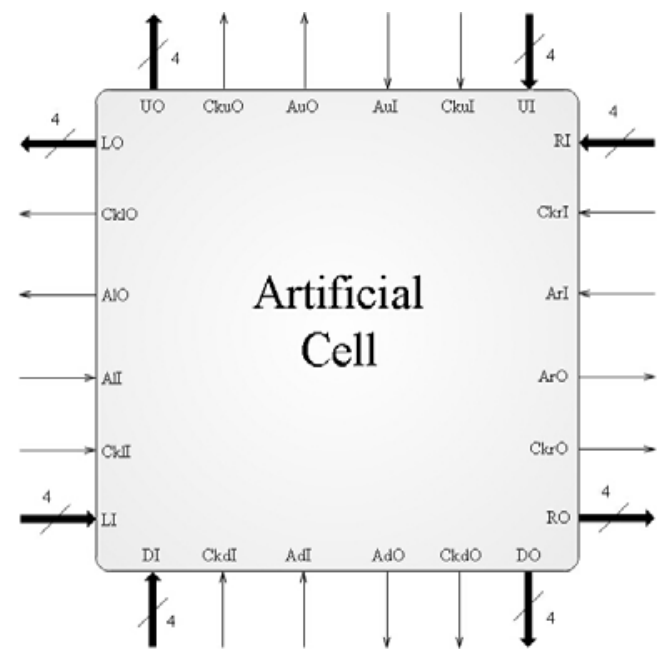

Fig. 2. Hardware model for FPGA-based artificial cell

Each lattice of the artificial cell has the same number of input/output bits and configuration structure, with the same operation functions. For example, the upper-lattice of the artificial cell has the following configuration:

- UI (b0ui, b1ui, b2ui, b3ui) - the data bus (4 input bits) to receive information from the upper neighbor cell;

- CkuI - the input data bus synchronization clock;
- UO (b0uo, b1uo, b2uo, b3uo) - the data bus (4 output bits) to send information to the upper neighbor cell;

- CkuO - the output data bus synchronization clock;

- AuI - signal received from the upper cell (alive=1, dead $=0$ )/data transaction enable signal;

- AuO - the cell's internal signal (alive=1, dead $=0$ )/data receive enable signal.

The same 12-bit configuration is used for the left, right, and down lattice of the artificial cell, and is specially designed for the full-operation of each FPGA circuit in a bi-dimensional artificial cell network [8].

Obviously, the proposed model is far not so complex; one artificial cell structure is suitable for convenient implementation also into one of the first generations Xilinx FPGA circuit. With the purpose to facilitate the operation algorithms for software implementation, the communication strategy between these cells is designed to work in a synchronous mode. It is wellknown, that now, the clock rate of several generation FPGA circuit's is up to $200 \mathrm{MHz}$. In this case, a maximal rate of $1 \mathrm{MHz}$ is limited, with the purpose to avoid inherent electrical noises and perturbations in the cells via-bus communication process. Moreover, for convenient visual monitoring of the cells interoperation, it is recommended, in the first laboratory experiments, to decrease this communication clock rate nearly up to 50$100 \mathrm{~Hz}$.

With the purpose of facilitating the research and modeling process too, the artificial cells are organized in macro-groups of cell networks, each with 9 artificial cells, as it is presented in figure 3 . In the software model indicated in this figure, each cell is an autonomous FPGA array, with special functions inside the organism, defined through an instruction set (program), and called the cell's gene. Each cell has a copy of all genes from the organism (operative genome), and depending on the cell's position inside the organism, only one cell has a gene operative (the cell's differentiation properties).

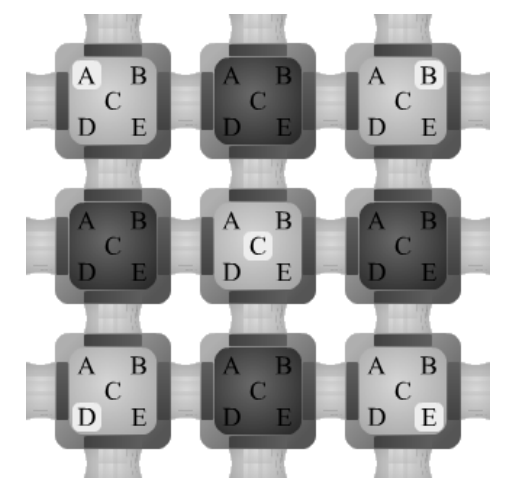

Fig.3. Model of artificial cell network (cluster)

In the presented model, the operative genome is designed with 5 genes $(A, B, C, D$, and $E)$, and at the same time just one gene is shown active (highlighted in the figure). The cells which don't show any operative gene are considered spare cells (4 cells from the total of 9 
in a cluster indicated in figure 3 ). It is important to note, that the implemented genes are generically labeled in the model with $A, B, C, D$, and $E$, but they can represent a wide range of control algorithms and programs (industrial process control, electrical motors control, etc.) defined by the software initialization process.

In accordance with the above mentioned strategy, in figure 4 an artificial organism model build with 4 macrogroup artificial cell network (clusters) is presented. In this model, a network with up to $40 \times 40=1600$ artificial cells is possible to be simulated. All cells in the artificial organism's structure are working according to the rules and communication abilities described in the FPGAbased model presented in chapter III.

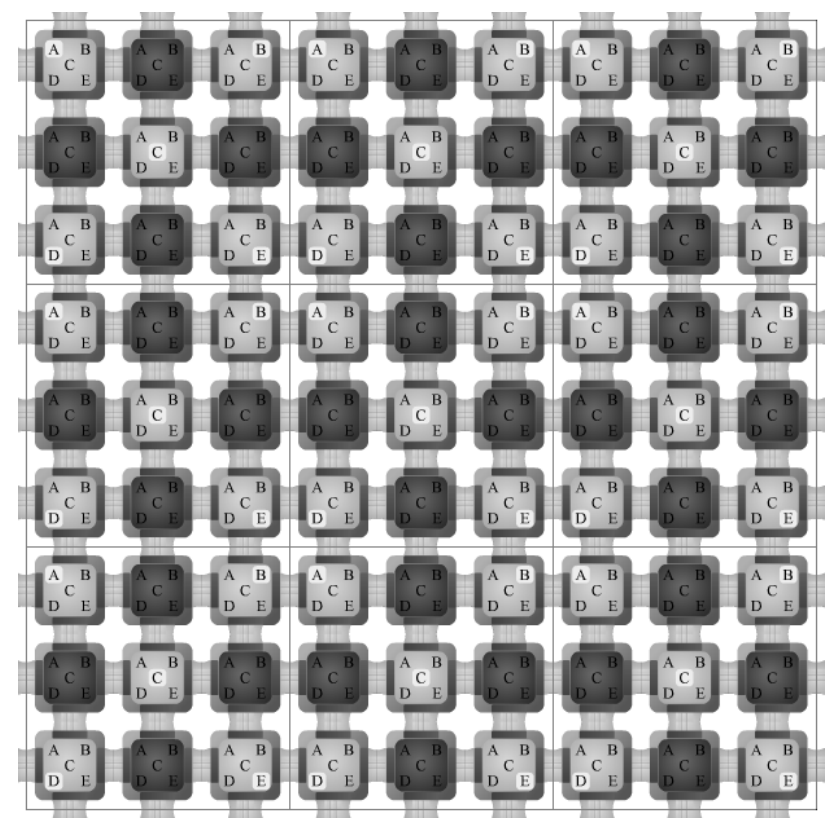

Fig. 4. Model of the artificial organism

The artificial organism represents the $3^{\text {rd }}$ level in the four level embryonic systems shown in figure 1 [9], [10].

\section{EMBRYONIC SYSTEMS COMPUTER-AIDED SIMULATION ENVIRONMENT}

In actual stage of modeling and simulation software environments development, the international corporations offer two main categories of simulation toolkits for the electronic circuit designers:

- simulation environments were the signals (voltages and currents) are continuous in the real time domain (for example: OrCAD PSpice);

- simulation environments were the signals (voltages and currents) can have only Boolean values (for example: Electonics Workbench Digital environment).

Simulation with continuous variables in the time domain is almost difficult, because needs accurate and complex computations, and is not suitable far away for real-time simulation of digital circuits operation. The simulation with Boolean variables seem to be the most simplest, and is suitable even for real-time simulation of complex VLSI digital circuits. In such a case the simulation is made through by the elementary circuits from the environment's library (logic gates, flip-flops, counters, multiplexers, etc.), or using the high level hardware description languages developed for this reason (for example: VHDL for ASIC's, FGPA's, etc.). But usually these kinds of environments are developed for simulation of one VLSI circuit, as the main part of the designed electronic circuit.

In special cases where is necessary the complex digital systems simulation, with several VLSI circuits in a network, these environments also becomes difficult an inefficient for utilization, especially for real-time simulation expectations.

Taking into account the above mentioned observations is not very difficult to deduce the following conclusion: for the embryonic models and FPGA-based artificial network structures presented in the previous paragraph, is not available in the market an adequate software simulation toolkit. This conclusion lead the team involved in the above mentioned embryonic hardware structures development to design a new simulation environment, specially conceived for complex VLSI circuit's network topologies real-time simulation. The main window of this simulation software toolkit is presented in figure 5 .

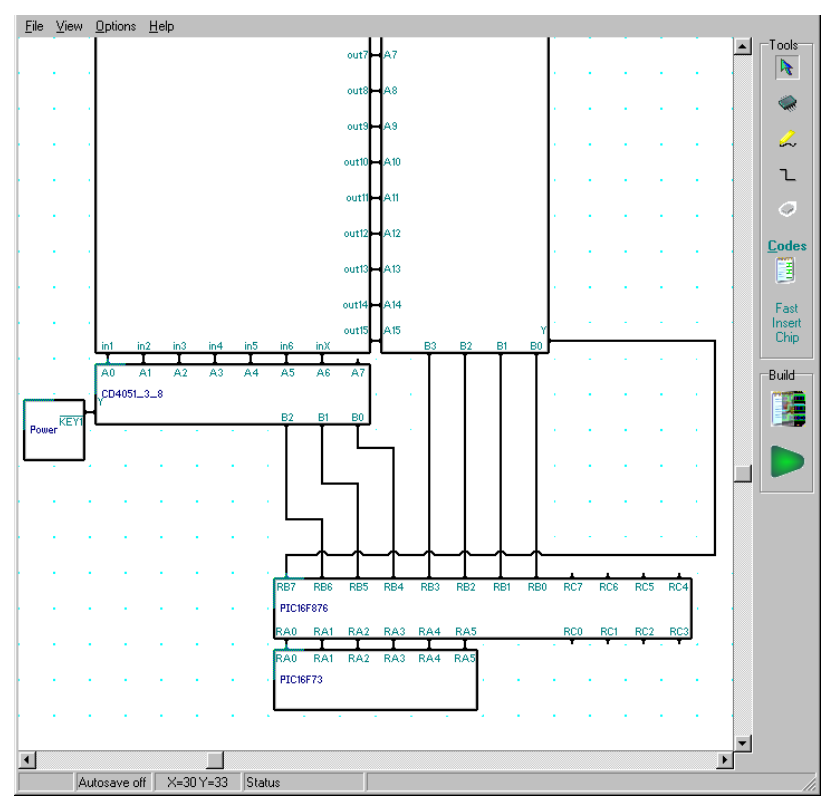

Fig. 5. Main window of the VLSI circuit's simulation software toolkit

The new simulation environment was conceived with a user-friendly interface, which allows the easy utilization and manipulation of the whole program. Figure 5 shows also the design pad where are pulled down the VLSI circuit's network. In accordance with the designer's expectations, de environment is able for real-time simulation of maximum 62500 digital circuits each with 8 pins, or a network with 25 VLSI circuits each own with maximum 400 pins. Of course, the hardware architecture 
of one complex circuit with 400 pins is implemented through VHDL language programming. In this reason, any kind of complex hardware can easily simulate in realtime operation, this depending only by the programmer's experience and its own implementation abilities.

To prove the new software toolkit generous simulation resources, in figure 6 a complex VLSI circuit's network is implemented on a design pad and prepared for real-time investigations.

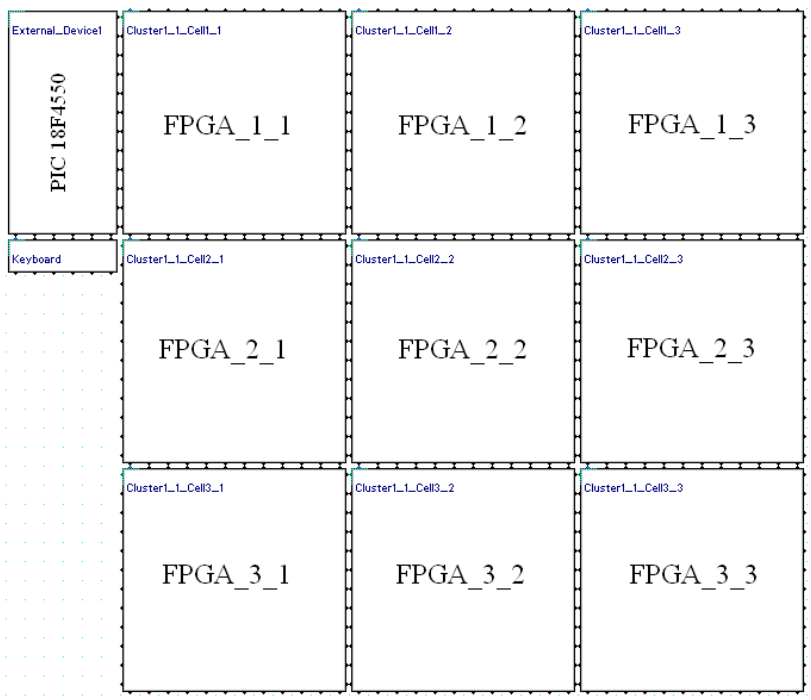

Fig. 6. Simulation model of the FPGA circuit's based artificial cell network organized in one cluster

The figure shows an artificial cell cluster, according to the software model presented in figure 3. Each cell from this network is an FPGA-based circuit, with the operation mode, external buses and hardware structure proposed in the artificial cell model from figure 2. Beside this all, the whole network is initialized and programmed through by a PIC 18F4550-type general purpose microcontroller via personal computer.

The whole software simulation package is divided into three main components: the unit for the VLSI circuit's edition and modeling, the module for the real-time simulation, and more one unit for the graphical elements simulation created by the user. Of course, a user-friendly software oscilloscope included into the toolkit is also available, for convenient real-time monitoring of the followed digital signals in the given embryonic system.

\section{COMUTER-AIDED SIMULATION RESULTS AND COMMUNICATION MONITORING}

Considering, that the FPGA circuit's based artificial cells communicate with each other via instructions in the network, defined through the communication strategy and rules described and explained in detail as follows in [7], at first, the artificial structure presented in figure 7 was considered for investigations and real-time simulations.

The basic goal of these simulations is to evidence the important facilities offered through by the new software environment, to demonstrate the versatility of programming in high level languages the operation mode of complex VLSI circuits, as it was proposed in the FPGA hardware model indicated in figure 2. Not at least, in the given case, to prove the right communication path inside of one artificial cell network (or cluster).

In this case, for more convenience an External Device able to generate excitation signals for the cell cluster is also connected in the structure. For example, it is used to send an instruction to Cell3_3 located in the right-down position of the cluster (with active gene $E$ ).

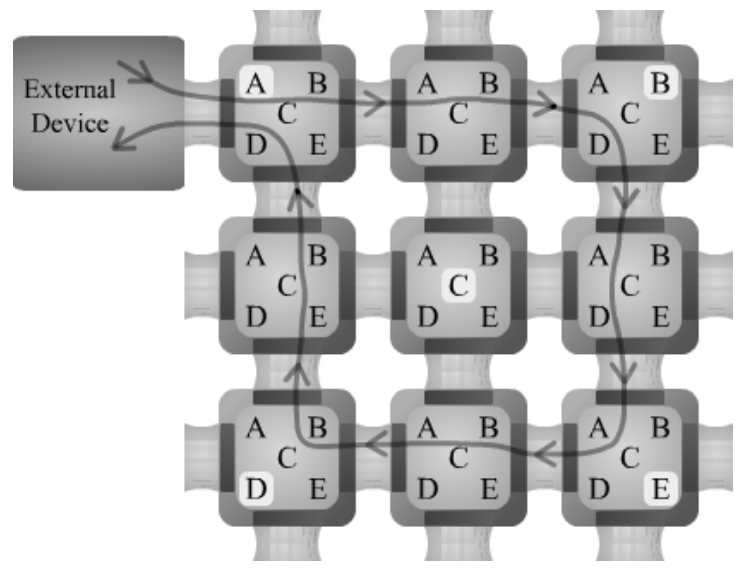

Fig. 7. Externally excited artificial cell network (example 1)

The obtained results can be followed in the timediagram presented figure 8 . At first, by the connected External Device the ExtDevice.Clk_o signal composed of 8 clocks (one instruction) is generated to the right-side cell (Cell1_1). This signal is received by Cell1_1 and through the Cell1_1.ckro is transmitted toward to the neighbor Cell1_2. In the same way the instruction is propagated in the cluster from one cell to other (the propagation path is marked through arrows). The Cell3_3 computes a result for the external device using gene $\bar{E}$, and at last Cell1_1 returns through Cell1_1.cklo this result to the External Device.

\begin{tabular}{|c|c|}
\hline External_Device1.Clk_o & 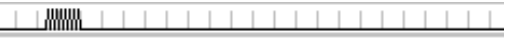 \\
\hline External_Device1.Clk_i & 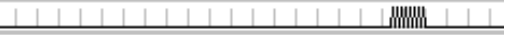 \\
\hline Cluster1_1_Cell1_1.ckli & 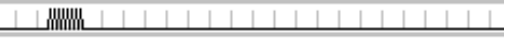 \\
\hline Cluster1_1_Cell1_1.ckro & 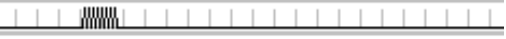 \\
\hline Cluster1_1_Cell1_2.ckli & 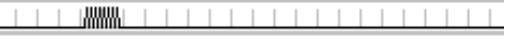 \\
\hline Cluster1_1_Cell1_2.ckro & 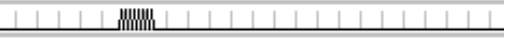 \\
\hline Cluster1_1_Cell1_3.ckli & 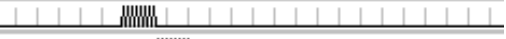 \\
\hline Cluster1_1_Cell1_3.ckdo & 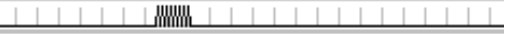 \\
\hline Cluster1_1_Cell2_1.ckdi & 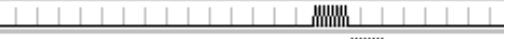 \\
\hline Cluster1_1_Cell2_1.ckuo & 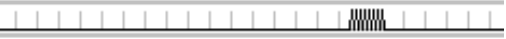 \\
\hline Cluster1_1_Cell2_3.ckui & 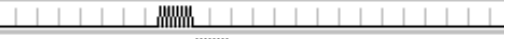 \\
\hline Cluster1_1_Cell2_3.ckdo & 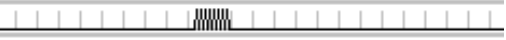 \\
\hline Cluster1_1_Cell3_1.ckri & 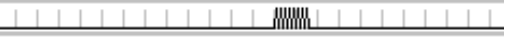 \\
\hline Cluster1_1_Cell3_1.ckuo & 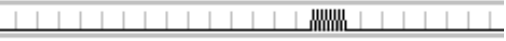 \\
\hline Cluster1_1_Cell3_2.ckri & 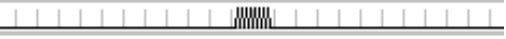 \\
\hline Cluster1_1_Cell3_2.cklo & 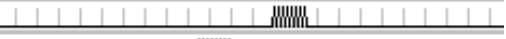 \\
\hline Cluster1_1_Cell3_3.ckui & 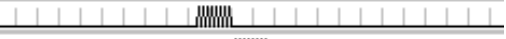 \\
\hline luster1_1_Cell3_3.cklo & 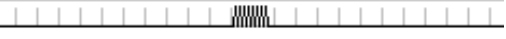 \\
\hline
\end{tabular}

Fig. 8. Network communication strategy inside the cluster (example 1)

The presented time-diagram evidences the delays of instructions between the artificial cells, and the right path of the communication. 
A new set of numerical simulation results can be obtained, if more complex embryonic system is proposed for computer-aided simulation and investigation.

In figure 9 is presented an artificial cell network structure (artificial organism) with the 36 artificial cells of 4 clusters. Now two External Devices are considered for the excitation signals generation: one in the upper-left side, and other in the down-left side of the network. Suppose that, they generate signals in the network as shows the two paths marked in the figure with arrows.

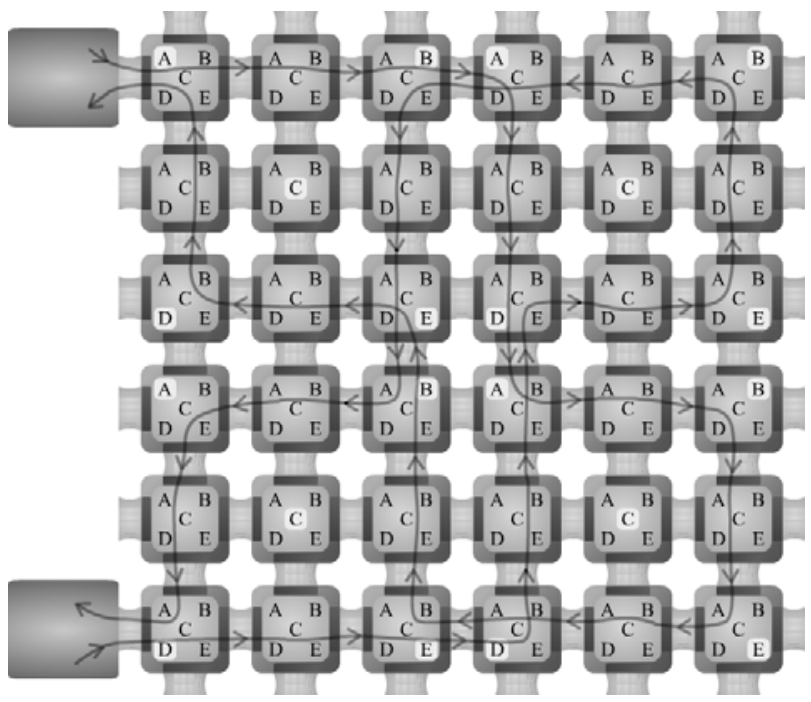

Fig. 9. Externally excited artificial cell network (example 2)

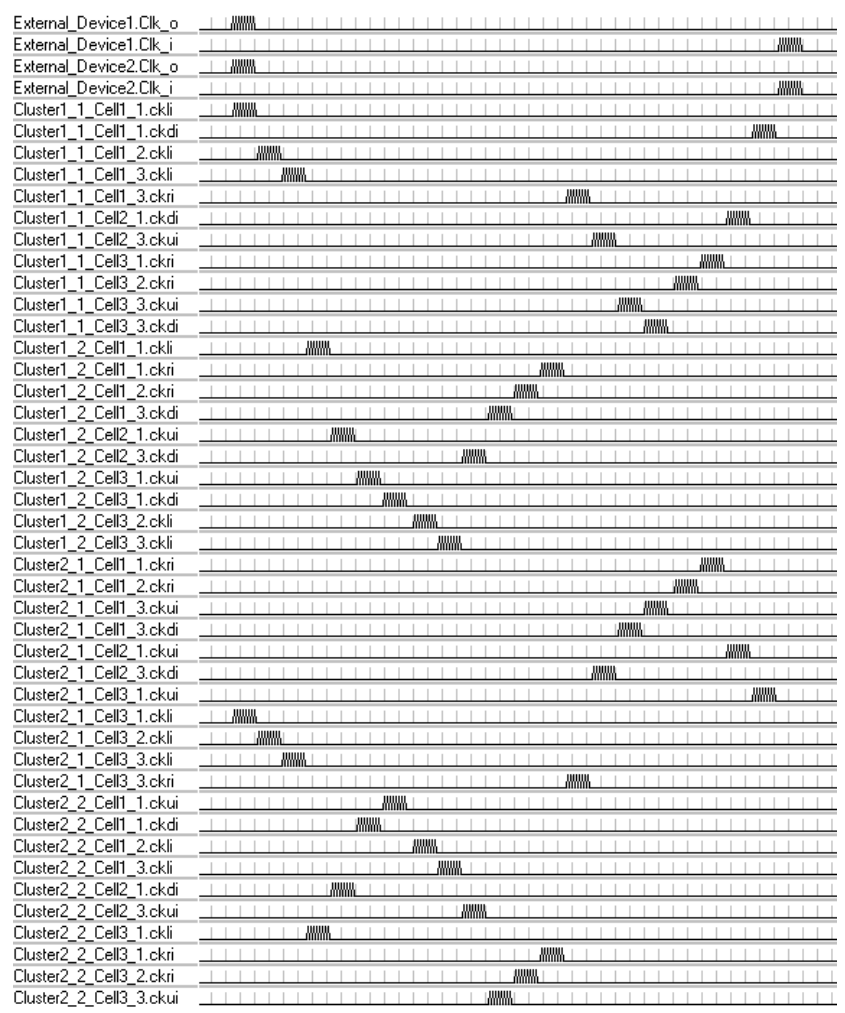

Fig. 10. Network communication strategy inside the cluster (example 2)

The obtained result is shown in the software oscilloscope from figure 10. Following the paths marked with arrows from figure 9 and the communication signals expressed in the left side of figure 10 , the right way of communication between the cells can be deduced.

From this point, the complexity of the modeled VLSI circuits (limited in the software toolkit to 25 circuits, each with a maximum of 400 connection pins) and the implemented algorithms (communication rules, genes implementation, etc.) length depends only by the experience and implementation abilities of the user.

\section{CONCLUSIONS}

In the paper artificial cell model and artificial organism models are proposed, as basic components of the four level POE-type embryonic structures. By adapting basic mechanisms and capabilities of living organisms from nature, is expected to engine complex novel digital systems and improve their capability, with the same robustness and fault-tolerance abilities like their biological equivalents.

Also a specially developed simulation software toolkit is presented; with the purpose of complex VLSI circuit's real-time simulation interconnected in network topologies. The proposed models were careful tested through careful computer-aided simulations.

The proposed models and artificial structures can be a support for future developments in bio-inspired hardware systems, in order to found the theoretical basis, the design models or development methods of this new science domain named the embryonic systems.

\section{Acknowledgement}

Work supported from the Romanian CNMP PNCDI2Partnership Research Grant ElBioArch, No. 12-121/2008.

\section{REFERENCES}

[1] Y. Thoma, G. Tempesti, E. Sanchez, J.M. Arostegni, "An electronic tissue for bio-inspired cellular applications" Biosystems, vol. 76, no. 1-3, pp. 191-200, August-October 2004.

[2] D. Mange, A. Stauffer, E. Petraglio, G. Tempesti, "Self-replication loop with universal construction" Physica D. vol. 191, no 1-2, pp. 178-192, April 2004.

[3] D. Mange, A. Stauffer, E. Petraglio, G. Tempesti, “Artificial cell division" Biosystems, vol. 76, no 1-3, pp. 157-167, Aug.-October 2004.

[4] A. Stauffer, D. Mange, G. Tempesti, "Bio-inspired computing machine with self-repair mechanism" Springer Berlin/Heidelberg, ISSN 0302-9743, vol. 3853, 2006.

[5] A. Upequi, Y. Thoma, E. Sanchez, A. Perez-uribe, J.M. Malho, J. Manderas, "The perplexus bio-inspired reconfigurable circuit" $2^{\text {nd }}$ NASA/ESA Conference on Adaptive Hardware and Systems (AHS), pp. 600-605, 2007.

[6] A. Stauffer, D. Mange, J. Rossier, "Design of self-organized bioinspired systems", $2^{\text {nd }}$ NASA/ESA Conference on Adaptive Hardware and Systems (AHS), pp. 413-419, 2007.

[7] Cs.Szász, V. Chindriş, "Artificial life and communication strategy in bio-inspired hardware systems with FPGA-based cell networks", $11^{\text {th }}$ IEEE International Conference on Intelligent Systems, 29 June - 1 July, Budapest Hungary, pp. 77-82, 2007

[8] Cs. Szász, V. Chindriş, L. Czumbil "Network communication strategy in embryonic systems with FPGA-based hardware", IEEE SMC International Conference on Distributed HumanMachine Systems, March 9-12, 2008 Athens, Greece, pp. 468473, 2008. 